\title{
BMJ Open Stool specimen for diagnosis of pulmonary tuberculosis in adults: protocol for a systematic review and meta-analysis
}

\author{
Saima Sultana (D) , ${ }^{1}$ Adnan Ansar (D) , ${ }^{2} \mathrm{~K}$ M Saif-Ur-Rahman (D) ${ }^{3,4}$
}

To cite: Sultana S, Ansar A, Saif-Ur-Rahman KM. Stool specimen for diagnosis of pulmonary tuberculosis in adults: protocol for a systematic review and meta-analysis. BMJ Open 2021;11:e052212. doi:10.1136/ bmjopen-2021-052212

- Prepublication history and additional supplemental material for this paper are available online. To view these files, please visit the journal online. (http://dx.doi.org/10.1136/ bmjopen-2021-052212)

Received 08 April 2021 Accepted 10 August 2021

Check for updates

(C) Author(s) (or their employer(s)) 2021. Re-use permitted under CC BY-NC. No commercial re-use. See rights and permissions. Published by BMJ.

${ }^{1}$ Projahnmo Research Foundation, Dhaka, Bangladesh ${ }^{2}$ School of Nursing and Midwifery, College of Science, Health and Engineering, La Trobe University, Melbourne, Victoria, Australia

${ }^{3}$ Public Health and Health Systems, Nagoya University Graduate School of Medicine Faculty of Medicine, Nagoya, Aichi, Japan

${ }^{4}$ Health Systems and Population Studies Division (HSPSD), icddr,b, Dhaka, Bangladesh

Correspondence to

Dr Saima Sultana;

saima.sultana.jhu@gmail.com

\section{ABSTRACT}

Introduction Tuberculosis (TB) continues to be a significant health burden, most commonly affecting the lungs and referred to as pulmonary TB (PTB). Diagnostic techniques of PTB primarily rely on expectorated sputum samples. However, the diagnostic yields are often hindered due to insufficient volume and quality of the sputum specimens. Moreover, some individuals are unable to provide sputum samples due to scanty sputum production or difficulty in coughing up and require an invasive procedure to obtain a respiratory sample, such as bronchoscopic or gastric aspiration. Thus, challenges in the acquisition of respiratory specimens warrant an alternate specimen. Therefore, this systematic review aims to evaluate the diagnostic accuracy of a stool specimen for the diagnosis of PTB in adults.

Methods and analysis We will search MEDLINE (Ovid), Embase (Ovid), Web of Science and Cochrane database from inception to April 2021 using a comprehensive search strategy. Two reviewers will independently perform screening, data extraction and quality assessment. The risk of bias assessment and applicability of results of eligible studies will be performed using the Quality of Diagnostic Accuracy Studies-2 tool. Bivariate random-effects models will be performed to calculate pooled sensitivity, specificity, positive likelihood ratio and negative likelihood ratio and diagnostic odds ratio along with $95 \% \mathrm{Cl}$ of stool specimen for each reported diagnostic method against any of the reference standard test (ie, mycobacterial culture or smear microscopy or Xpert assay using respiratory specimens). Heterogeneity between studies will be assessed by $I^{2}$ statistics and $Q$ statistic of the $\chi^{2}$ test.

Ethics and dissemination The results will be disseminated through publishing in a peer-reviewed medical journal and public presentations in relevant national and international conferences. As this is a systematic review of publicly available data, ethics approval is not required.

PROSPERO registration number CRD42021245203.

\section{INTRODUCTION}

Tuberculosis (TB) is an infectious bacterial disease caused by Mycobacterium tuberculosis (MTB). It remains one of the top 10 causes of death in lower-income and lower-middleincome countries. ${ }^{12}$ According to the 2020
Strengths and limitations of this study

This is the first systematic review and meta-analysis that will synthesise the evidence on the diagnostic performance of stool specimens for the diagnosis of pulmonary tuberculosis in adults.

- This systematic review will include all diagnostic techniques (microscopy, culture and PCR assay, including Xpert) that would be helpful to identify the best diagnostic option for stool specimens.

- Stringent methodologies and robust analysis will be employed to minimise the risk of bias and report accurate data.

- There might be significant heterogeneity among the studies that would limit the interpretation of results.

Global Tuberculosis Report, in 2019, there was an estimated 10 million new TB cases, with approximately 1.4 million deaths due to TB. ${ }^{3}$ TB is an airborne disease that is mainly transmitted between humans through respiratory droplets generated during coughing, sneezing or speaking. It most commonly affects the lungs, known as pulmonary TB (PTB) but can also involve extrapulmonary sites in the body, including-lymph nodes, pleura, abdomen, urogenital tract, skin, joints and bones and meninges. ${ }^{4}$ Bacteriological confirmation of PTB usually depends on microscopy, culture and PCR-based assay, including Xpert assay using sputum specimens. However, the diagnostic performance of these tests depends on the concentration of MTB in sputum samples. Poor quality and inadequate volume of sputum samples can lead to missed diagnoses, delays in initiating treatment and increased risk of transmission to others from undiagnosed or untreated individuals. ${ }^{56}$ In addition, at least two sputum specimens (one spot specimen at the time of initial consultation followed by one early morning specimen on the next day) are recommended, which can entail additional 
costs and inconvenience to individuals due to repeated visits to a healthcare facility. ${ }^{6}$ Furthermore, some individuals may have found it challenging to provide an expectorated sputum sample, especially young children, the elderly, severely ill, people living with human immunodeficiency virus or pregnant women. In these situations, induced sputum, bronchoalveolar lavage fluid or gastric lavage fluid are used as alternative diagnostic specimens. However, procedures for obtaining these specimens are invasive and impose additional costs; they also require technical expertise that may not be readily available in resource-constrained settings. ${ }^{7-9}$

Diagnostic testing of stool samples is an alternative to respiratory specimens for diagnosis of PTB. As sputum is swallowed and MTB passes through the digestive tract, it can be detected in stool through microscopy, culture and PCR tests, including Xpert assay. ${ }^{10}{ }^{11}$ Recent studies on PTB diagnosis using the Xpert platform on stool samples have shown promising results, and this approach has been increasingly used in the paediatric population for PTB diagnosis. ${ }^{12}$ While some studies on the use of stool samples for PTB diagnosis in adults have been published, its accuracy and utility compared with standard diagnostic approaches have not yet been evaluated through a systematic review. It is also important to recognise the best method that would provide maximum diagnostic accuracy to detect PTB using the stool samples. Therefore, this systematic review aims to evaluate the diagnostic accuracy of stool specimens in microscopy, culture and PCR assays to diagnose PTB in adults against any microbiological reference standard tests, that is, smear microscopy or culture or Xpert assays using respiratory specimens. The results would provide crucial evidence in the TB diagnostic landscape in adults, particularly those who cannot expectorate sputum or produce inadequate sputum.

\section{METHODS AND ANALYSIS}

This systematic review will be conducted and reported following the Preferred Reporting Items for Systematic Reviews and Meta-Analyses of Diagnostic Test Accuracy (PRISMA-DTA) criteria (see online supplemental files S1 and S2 for PRISMA-DTA checklists). ${ }^{13}$ In addition, the results of the literature search and screening will be presented in a PRISMA flow diagram. ${ }^{14}$

\section{Search strategy and information sources}

A comprehensive search strategy will be developed to search relevant studies in the following electronic bibliographical databases; MEDLINE (Ovid) (1946-April 2021), Embase (Ovid) (1947-April 2021), Web of Science (1950-April 2021) and Cochrane database (inceptionApril 2021). To develop the database search strategies, we will use Medical subject headings (MeSH) as well as terms, keywords, and synonyms such as "Mycobacterium tuberculosis" or "MTB," "pulmonary tuberculosis" or "PTB," "tuberculosis," or "TB," "adults" or "elderly," "stool" or "faeces" or "faecal," and "diagnosis" or "diagnostic" (Table 1; see online supplemental file S3 for the search strategy). In addition, we will search references cited in the included articles to avoid missing relevant studies. When required, we will also communicate with study authors for further information. No restrictions will be applied on the publication dates, publication status and study designs. We will not search for unpublished studies or grey literature.

Table 1 presents the search strategy for MEDLINE (Ovid) and Embase (Ovid).

\section{Selection criteria}

Studies will be identified based on the following inclusion criteria: (1) evaluated stool specimen in adults (aged 18 years and older) with presumptive/active PTB using microscopy or culture or PCR assay, including Xpert (index test); (2) diagnosis of PTB in study participants was accompanied by bacteriological confirmation of MTB in the respiratory specimen by culture and/or microscopic examination, and/or Xpert assay (reference test); (3) type of studies: both retrospective and prospective crosssectional and cohort studies, randomised controlled trials and case-control studies that used stool samples for PTB diagnosis; (4) studies that assessed diagnostic accuracy and/or provided sufficient data to compute diagnostic accuracy measures ((true positive (TP), false positive (FP), true negative (TN), false negative $(\mathrm{FN})$ ); (5) studies that used stored/banked sputum and stool specimen for analysis will also be eligible; (6) studies that included both adults and children provided that disaggregated adult data is available.

Studies are not eligible if (1) no study participants 18 years of age or older; (2) stool specimen was not tested for PTB diagnosis; (3) reviews, conference proceedings and abstracts, case reports, editorials and commentaries and (4) articles in languages other than English.

\section{Data management}

Results from the search of the bibliographic databases will be managed using Covidence, a web-based platform developed by the Cochrane Collaboration that supports the synthesis of evidence for systematic reviews. ${ }^{15}$ All articles will be imported into Covidence, and duplicate articles will be identified and removed accordingly.

\section{Study screening and data abstraction}

Initially, screening of the title and abstract of the retrieved articles will be conducted by two reviewers independently according to the predetermined study selection criteria using the Covidence platform. After initial screening, the full-text articles will be sought for potentially eligible studies, with the assessment of eligibility conducted by two reviewers independently. Any inconsistencies between two reviewers at any stage of the review process will be resolved through discussion or consultation with a third reviewer. 
Table 1 MEDLINE (Ovid) and Embase (Ovid) search strategy

\begin{aligned} \hline$\# &$ Searches \\ \hline 1 & exp Mycobacterium tuberculosis/ \\ 2 & (Mycobacterium tuberculosis or MTB or \\ & mycobacterium tuberculosis complex).mp. [mp=title, \\ & abstract, original title, name of substance word, \\ & subject heading word, floating sub-heading word, \\ & keyword heading word, organism supplementary \\ & concept word, protocol supplementary concept word, \\ & rare disease supplementary concept word, unique \\ & identifier, synonyms] \end{aligned}

3 exp Tuberculosis/

4 exp Tuberculosis, Pulmonary/

5 (TB or PTB or pulmonary tuberculosis or lung tuberculosis).mp. [mp=title, abstract, original title, name of substance word, subject heading word, floating sub-heading word, keyword heading word, organism supplementary concept word, protocol supplementary concept word, rare disease supplementary concept word, unique identifier, synonyms]

61 or 2 or 3 or 4 or 5

7 exp Adult/

8 exp Aged/

9 exp "Aged, 80 and over"/

10 exp Middle Aged/

11 (aged or elder ${ }^{\star}$ or adult*).mp. [mp=title, abstract, original title, name of substance word, subject heading word, floating sub-heading word, keyword heading word, organism supplementary concept word, protocol supplementary concept word, rare disease supplementary concept word, unique identifier, synonyms]

127 or 8 or 9 or 10 or 11

13 exp Feces/

14 (stool or f?eces or f?ecal).mp. [mp=title, abstract, original title, name of substance word, subject heading word, floating sub-heading word, keyword heading word, organism supplementary concept word, protocol supplementary concept word, rare disease supplementary concept word, unique identifier, synonyms]

15 ((stool or f?eces or f?ecal) adj3 (analysis or sample* or specimen $\left.{ }^{\star}\right)$ ).mp. [mp=title, abstract, original title, name of substance word, subject heading word, floating sub-heading word, keyword heading word, organism supplementary concept word, protocol supplementary concept word, rare disease supplementary concept word, unique identifier, synonyms]

$\begin{array}{ll}16 & 13 \text { or } 14 \text { or } 15 \\ 17 & \exp \text { Diagnosis/ } \\ 18 & \exp \text { "Diagnostic Techniques and Procedures"/ } \\ 19 & \text { exp "Sensitivity and Specificity"/ } \\ 20 & \text { diagnos*.mp. }\end{array}$

Continued

\begin{tabular}{|c|c|}
\hline$\#$ & Searches \\
\hline 21 & $\begin{array}{l}\text { (diagnos }{ }^{*} \text { adj3 (accuracy or performance)).mp. } \\
\text { [mp=title, abstract, original title, name of substance } \\
\text { word, subject heading word, floating sub- } \\
\text { heading word, keyword heading word, organism } \\
\text { supplementary concept word, protocol supplementary } \\
\text { concept word, rare disease supplementary concept } \\
\text { word, unique identifier, synonyms] }\end{array}$ \\
\hline 22 & 17 or 18 or 19 or 20 or 21 \\
\hline 23 & 6 and 12 and 16 and 22 \\
\hline
\end{tabular}

A data extraction form will be created using an Excel spreadsheet and piloted on three eligible studies. Two reviewers will independently extract data from each selected article and complete the data extraction form that will consist of the following data items:

- General information: study title, author(s), year of publication, study country, including the WHO classification for TB burden country (ie, high TB burden or low TB burden countries), study settings, study design.

- Summary statistics on age, gender, HIV status, other comorbid conditions

- Sample size.

- Case definitions and reference standard test/s for PTB diagnosis.

- Index test/s (ie, microscopy or culture or PCR assays, including Xpert on stool samples).

- Type of specimen (eg, stool, sputum, induced sputum, bronchoalveolar lavage, gastric aspirate).

- Volume of specimen.

- Specimen condition (fresh vs stored/frozen).

- Specimen processing method.

- Timing of tests (ie, the interval between the index and reference standard test/s).

- Outcome measures: numbers of TP, FP, FN and TN against the reference standard tests.

In the case of multiple reference standard tests within the same study, the results will be recorded separately.

\section{Quality assessment}

Risk of bias assessment and applicability of results of included studies will be performed using the Quality of Diagnostic Accuracy Studies-2 (QUADAS-2), a recommended tool for appraising studies in systematic reviews for diagnostic accuracy. ${ }^{16}$ As per QUADAS-2 guideline, two reviewers will independently assess the risk of bias of each included study in four key domains: (a) patient selection, (b) index test, (c) reference standard, and (d) flow of patients and timing of index and reference standard tests. The risk of bias or applicability concerns will be qualified as 'low,' 'high' or 'unclear. ${ }^{16}$ Similar to article selection phases, disagreement between reviewers will be solved by discussion or consultation with a third reviewer. 


\section{Data analysis}

Initially, we will perform a narrative synthesis of all included studies and summarise the results, including characteristics of included studies and participants, sample type for reference standard test/s, and details of sample processing and storage methods.

Meta-analysis will be performed using the MIDAS module in STATA statistical software (V.16.0, Stata). We will construct $2 \times 2$ tables for all included studies and enter TP, FP, FN and $\mathrm{TN}$ for all index test/s against the reference standard test/s. If necessary, additional data may be sought from the study corresponding author through email to support meta-analysis. If this is unsuccessful, studies will be omitted from the pooled meta-analysis. We will use bivariate randomeffects models to calculate pooled sensitivity, specificity, positive likelihood ratio and negative likelihood ratio and diagnostic odds ratio along with $95 \%$ CI of stool specimen for each reported diagnostic method (ie, microscopy, culture or PCR assay) for diagnosis of PTB against smear microscopy or culture or Xpert assay on a respiratory specimen. We will also report the sensitivity and specificity of each study in forest plots and analyse the areas under the summary receiver operating characteristic curves. If there is adequate data, subgroup analysis (eg, HIV status, stool processing method, conditions of the specimen (fresh or frozen/stored)) and meta-regression analysis will be performed. If deemed appropriate, sensitivity analysis will be done to evaluate the effect of risk of bias by excluding the studies of lower methodological quality (ie, high or uncertain risk of bias).

We will summarise the key results in the 'Summary of Findings' tables and assess the certainty of evidence using the Grading of Recommendations Assessment, Development and Evaluation (GRADE) guideline for diagnostic tests. ${ }^{17}$

\section{Assessment of heterogeneity and publication bias}

We will estimate the heterogeneity between the studies by using $\mathrm{I}^{2}$ statistics and $Q$ statistic of the $\chi^{2}$ test. If possible, the source of heterogeneity will be examined by conducting subgroup analysis or meta-regression. Potential publication bias will be assessed using Deeks test. ${ }^{18}$

\section{Patient and public involvement}

The patient and the public were not involved during the conceptualisation and development of the review protocol. However, we intend to involve the patients during the dissemination of findings in national conferences.

\section{Start date}

15 April 2021.

\section{Anticipated completion date}

31 October 2021.

\section{ETHICS AND DISSEMINATION}

Ethics approval will not be required for this study protocol as this systematic review focuses on the analysis of published data. On completing the review, we will disseminate the results in a peer-reviewed medical journal and present them at relevant conferences.

\section{DISCUSSION}

Rapid and accurate diagnosis of PTB is crucial for timely initiation of treatment, preventing transmission and improving the prognosis of individuals with PTB. However, diagnosing PTB can be challenging due to difficulty in obtaining sputum specimens, especially those who are unable to produce sputum. Thus, there is a need for an alternative, non-sputum-based sample for PTB diagnosis. To the best of our knowledge, this will be the first systematic review that will evaluate the diagnostic accuracy of the stool specimens for PTB diagnosis in adults. We anticipate that the results of this review will support clinicians and policymakers to provide guidance in clinical laboratory practice for the diagnosis of PTB in adults. It will also guide future research needs based on identified gaps and alleviate the pathway to end TB endemic across the globe.

The strength and limitations of the included studies will be discussed. We will assess the strength of the body of evidence and highlight the gaps in the evidence for future research, using the GRADE approach for diagnostic accuracy studies.

Acknowledgements We would like to acknowledge Joshua Vogel for English language editing. In addition, the author (KMS-U-R) would like to acknowledge the contribution of the current donors providing unrestricted support to icddr,b that include: the Governments of Bangladesh, Canada, Sweden and the UK. We gratefully acknowledge these donors for their support and commitment to icddr,b's research efforts.

Contributors SS conceptualised, designed and drafted the initial manuscript. SS and KMS-U-R developed the search strategy. AA and KMS-U-R critically apprised and edited the manuscript. All authors reviewed the manuscript and have approved the final manuscript before submission. SS is the guarantor of this review.

Funding The authors have not declared a specific grant for this research from any funding agency in the public, commercial or not-for-profit sectors.

Competing interests None declared.

Patient consent for publication Not required.

Provenance and peer review Not commissioned; externally peer reviewed.

Supplemental material This content has been supplied by the author(s). It has not been vetted by BMJ Publishing Group Limited (BMJ) and may not have been peer-reviewed. Any opinions or recommendations discussed are solely those of the author(s) and are not endorsed by BMJ. BMJ disclaims all liability and responsibility arising from any reliance placed on the content. Where the content includes any translated material, BMJ does not warrant the accuracy and reliability of the translations (including but not limited to local regulations, clinical guidelines, terminology, drug names and drug dosages), and is not responsible for any error and/or omissions arising from translation and adaptation or otherwise.

Open access This is an open access article distributed in accordance with the Creative Commons Attribution Non Commercial (CC BY-NC 4.0) license, which permits others to distribute, remix, adapt, build upon this work non-commercially, and license their derivative works on different terms, provided the original work is properly cited, appropriate credit is given, any changes made indicated, and the use is non-commercial. See: http://creativecommons.org/licenses/by-nc/4.0/.

ORCID iDs

Saima Sultana http://orcid.org/0000-0001-6129-918X

Adnan Ansar http://orcid.org/0000-0001-6052-8657

K M Saif-Ur-Rahman http://orcid.org/0000-0001-8702-7094 


\section{REFERENCES}

1 Bloom BR, Atun R, Cohen T. Tuberculosis. In: Holmes KK, Bertozzi $\mathrm{S}$, Bloom BR, eds. Major infectious diseases. Washington (DC: The International Bank for Reconstruction and Development / The World Bank (C) 2017 International Bank for Reconstruction and Development / The World Bank, 2017.

2 World health statistics 2021: a visual summary: World Health organization, 2021. Available: https://www.who.int/data/stories/ world-health-statistics-2021-a-visual-summary [Accessed 17 Jun 2021].

3 WHO. Global tuberculosis report 2020. Geneva: World Health Organization, 2020.

4 Sharma SK, Mohan A, Kohli M. Extrapulmonary tuberculosis. Expert Rev Respir Med 2021.

5 Datta S, Shah L, Gilman RH, et al. Comparison of sputum collection methods for tuberculosis diagnosis: a systematic review and pairwise and network meta-analysis. Lancet Glob Health 2017;5:e760-71.

6 Parsons LM, Somoskövi A, Gutierrez C, et al. Laboratory diagnosis of tuberculosis in resource-poor countries: challenges and opportunities. Clin Microbiol Rev 2011;24:314-50.

7 Luo W, Lin Y, Li Z, et al. Comparison of sputum induction and bronchoscopy in diagnosis of sputum smear-negative pulmonary tuberculosis: a systemic review and meta-analysis. BMC Pulm Med 2020;20:146.

8 Ruiz Jiménez M, Guillén Martín S, Prieto Tato LM, et al. "Induced sputum versus gastric lavage for the diagnosis of pulmonary tuberculosis in children". BMC Infect Dis 2013;13:222.

9 Tan H-K, Fan S-J, Xu Y-C, et al. The clinical diagnostic value of Xpert MTB/RIF for the detection of Mycobacterium tuberculosis in gastric aspirates. Biosci Rep 2020;40:BSR20200138.
10 Donald PR, Schaaf HS, Gie RP, et al. Stool microscopy and culture to assist the diagnosis of pulmonary tuberculosis in childhood. $J$ Trop Pediatr 1996;42:311-2.

11 Walters E, Demers A-M, van der Zalm MM, et al. Stool culture for diagnosis of pulmonary tuberculosis in children. J Clin Microbiol 2017:55:3355-65.

12 MacLean E, Sulis G, Denkinger CM, et al. Diagnostic accuracy of stool Xpert MTB/RIF for detection of pulmonary tuberculosis in children: a systematic review and meta-analysis. J Clin Microbiol 2019;57:e02057-18.

13 Salameh J-P, Bossuyt PM, McGrath TA, et al. Preferred reporting items for systematic review and meta-analysis of diagnostic test accuracy studies (PRISMA-DTA): explanation, elaboration, and checklist. BMJ 2020;370:m2632.

14 Page MJ, McKenzie JE, Bossuyt PM, et al. The PRISMA 2020 statement: an updated guideline for reporting systematic reviews. BMJ 2021;372:n71.

15 Covidence systematic review software. Melbourne, Australia: Veritas Health Innovation 2017.

16 Whiting PF, Rutjes AWS, Westwood ME, et al. QUADAS-2: a revised tool for the quality assessment of diagnostic accuracy studies. Ann Intern Med 2011;155:529-36.

17 Schünemann HJ, Schünemann AHJ, Oxman AD, Brozek J, et al. Grading quality of evidence and strength of recommendations for diagnostic tests and strategies. BMJ 2008;336:1106-10.

18 Deeks JJ, Macaskill P, Irwig L. The performance of tests of publication bias and other sample size effects in systematic reviews of diagnostic test accuracy was assessed. J Clin Epidemiol 2005;58:882-93. 